Case

Report

\title{
A 3.5-cm Single-Incision VATS Anatomical Segmentectomy for Lung Cancer
}

\author{
Mitsuhiro Kamiyoshihara, MD, PhD, ${ }^{1}$ Hitoshi Igai, MD, PhD,${ }^{1}$ Takashi Ibe, MD, PhD, ${ }^{1}$ \\ Natsuko Kawatani, MD, ${ }^{1}$ Kimihiro Shimizu, $\mathrm{MD}, \mathrm{PhD},{ }^{2}$ and Izumi Takeyoshi, $\mathrm{MD}, \mathrm{PhD}^{2}$
}

\begin{abstract}
The results of several pulmonary resections using a uniportal approach have been published. However, there are no reports of uniportal thoracoscopic anatomic segmentectomy in Japan. We have a fundamental belief in "reduced-port surgery" and therefore routinely perform uniportal thoracoscopic surgery for patients with pneumothorax. This report describes a successful case of uniportal thoracoscopic anatomic segmentectomy through a $3.5-\mathrm{cm}$ incision in a 76-year-old woman with primary lung cancer. The patient was pathologically diagnosed with multiple primary adenocarcinomas stage IA (T1aN0M0). Postoperatively, no analgesics were needed. The operative procedure is described in detail and includes technical tips such as the pulley method, extra-vessel exposure, the shaft-on-shaft technique, one-hand encircling, and one-hand exposure. The selection criteria for uniportal thoracoscopic segmentectomy limit its use.
\end{abstract}

Keywords: pulmonary segmentectomy, single incision, thoracoscopic surgery

\section{Introduction}

Generally, total thoracoscopic pulmonary segmentectomy is performed through more than three incisions. Since Gonzalez-Rivas, et al. ${ }^{1)}$ first described uniportal video-assisted thoracoscopic surgery (VATS) for anatomic segmentectomy, two additional groups have published the results of anatomic segmentectomy through a uniportal approach. ${ }^{2,3)}$ Uniportal VATS for anatomic segmentectomy is a complex procedure because the segmental planes need to be identified for pulmonary segmentation, making the procedure more technically challenging than

${ }^{1}$ Department of General Thoracic Surgery, Maebashi Red Cross Hospital, Maebashi, Gunma, Japan

${ }^{2}$ Division of Thoracic and Visceral Organ Surgery, Gunma University

Graduate School of Medicine, Maebashi, Gunma, Japan

Received: June 22, 2014; Accepted: July 26, 2014

Corresponding author: Mitsuhiro Kamiyoshihara, MD, $\mathrm{PhD}$. Department of General Thoracic Surgery, Maebashi Red Cross Hospital, 3-21-36 Asahi-Cho, Maebashi, Gunma 371-0014, Japan Email: micha2005jp@yahoo.co.jp

(C)2014 The Editorial Committee of Annals of Thoracic and Cardiovascular Surgery. All rights reserved. a lobectomy. However, there are no reports of uniportal VATS for segmentectomy in Japan. Our institute performs uniportal thoracoscopic surgery for pneumothorax due to a fundamental belief of "reduced-port surgery."4) This report describes a successful case of uniportal VATS anatomic segmentectomy through a $3.5-\mathrm{cm}$ incision.

\section{Case Report}

Thirty-nine months ago, a 76-year-old female patient underwent conventional three-port VATS for pulmonary segmentectomy including a right upper lobectomy, superior segmentectomy (segment 6) of the right lower lobe, and wedge resection of the medial segment (segment 5) of the right middle lobe for multiple primary pulmonary adenocarcinomas (stage IB [pT2aN0M0] in right upper lobe, stage IA [pT1bNOM0] in segment 6 , and stage IA [pT1aN0M0] in segment 5). The malignant tumors were completely resected (R0).

Follow-up included a computed tomography (CT) scan every 6 months. Two years after the initial operation, a follow-up CT scan (Fig. 1A) demonstrated four nodules of ground glass opacity (GGO) that had gradually grown. 

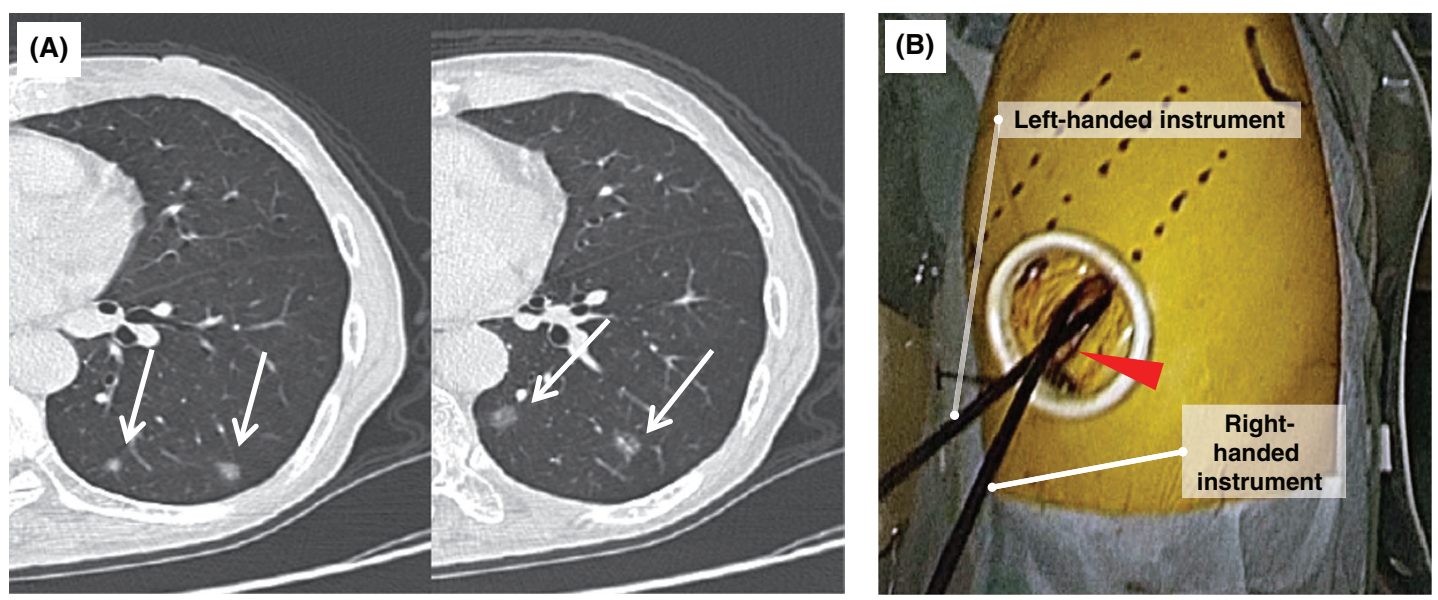

Fig. 1 (A) Computed tomography demonstrates multiple nodules of ground glass opacity in the basal segment of the left lower lobe. (B) Exterior appearance. Instrumentation for uniportal video-assisted thoracoscopic surgery segmentectomy. The shaft of a right-handed instrument (e.g., scissors, an energy device, or a vacuum) was held against the shaft of a left-handed instrument (e.g., a grasper or a sponge stick) at the edge of the incision. Note that a pivot point was created using the right-handed instrument in a crossed-shaft position (arrowhead).

All four nodules, including one 6-mm, one 8-mm, and two 11-mm nodules, were located in the basal segment of the left lower lobe. These findings suggested a multiple primary lung cancer rather than recurrence of previous lung cancer based on the previous postoperative pathology and the appearance of GGO. 18F-fluorodeoxyglucose positron emission tomography-CT (PET-CT) demonstrated that hilar and mediastinal lymph nodes were negative for metastasis.

Surgical rather than medical treatment was chosen despite the presence of an epidermal growth factor receptor mutation in the tumor, because of the patient's preference for surgical intervention and well-preserved pulmonary function. Pulmonary function tests revealed a vital capacity of $1.2 \mathrm{~L}(55 \%)$ and a forced expiratory volume in $1 \mathrm{~s}$ of $1.2 \mathrm{~L}(100 \%)$.

In general, the instrumentation of conventional VATS is also used for uniportal VATS. Instruments are preferably long and curved to allow for simultaneous insertion of two or three instruments. In addition, a 5-mm flexible thoracoscope (HD Endo EYE, LTF-VH; Olympus, Tokyo, Japan) ${ }^{5)}$ and an energy device (ENSEAL ${ }^{\circledR}$ TRIO; Ethicon Endo-Surgery, Cincinnati, Ohio, USA) are used during the surgery. ${ }^{6}$ In the present case, the patient, under general anesthesia with double-lumen intubation, was placed in the full lateral decubitus position with slight flexion of the table at the level of the mid-chest. The operator and the thoracoscope assistant stood on the anterior and posterior side of the patient, respectively. The scope was placed on the posterior side and the other working instruments were placed on the anterior side. When needed, the surgical table was rolled forward or backward to secure the operative field.

A $3.5-\mathrm{cm}$ incision was made in the sixth intercostal space on the anterior axillary line (Fig. 1B). The incision was protected with a polyurethane wound retractor (Alexis wound retractor; Applied Medical, Rancho Santa Margarita, California, USA).

After deflating the lung, the pulmonary basal artery and vein were defined and divided with an articulated endoscopic stapler. We arbitrarily bend an articulated stapler to assist with stapler placement. We achieve optimal angles for stapler insertion into vessels with adequate exposure. We retracted the piece of silicone tape encircling the vessel across the pulmonary vessel to facilitate insertion of the stapler when there is a difficult angle (Fig. 2A). We prefer an Endo GIA ${ }^{\mathrm{TM}}$ curved tip with a gray-colored, 30-mm-long stapler (Endo GIA ${ }^{\mathrm{TM}}$ Ultra Universal and Curved Tip Articulating Vascular Cartridge; Covidien Surgical, Norwalk, Connecticut, USA) for the approach to pulmonary vessels rather than a conventional straight stapler for uniportal VATS. This avoids damage to the back or the other side of the vessel in a limited space because the curved tip has a slim and forward-jutting jaw. Additionally, adequate exposure of the vessel periphery facilitates insertion of the stapler because the insertion angle of the stapler increases (Fig. 2B). 

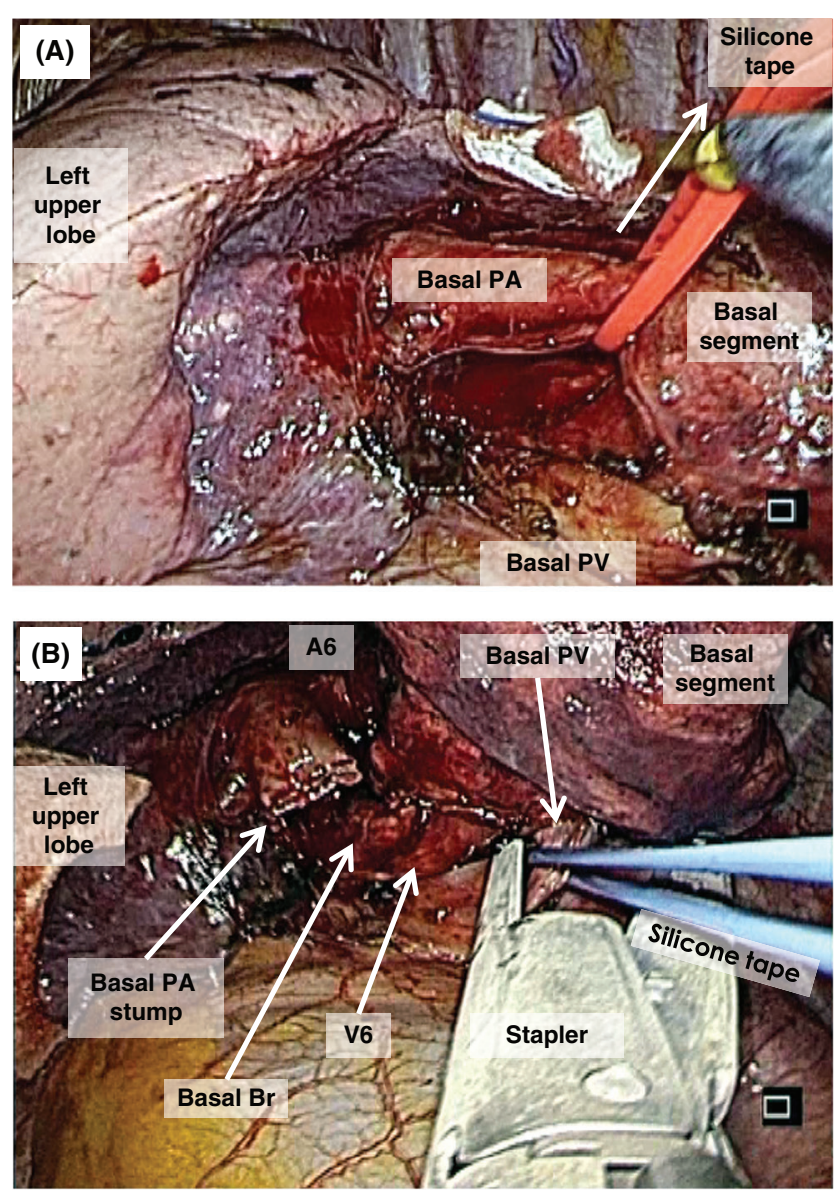

Fig. 2 (A) The pulley method: We achieved optimal angles for inserting a stapler into vessels when we had adequate exposure. We retract (arrow's direction) a piece of silicone tape encircling the vessel across the pulmonary vessel or bronchus to facilitate insertion of the stapler when there is a difficult angle. (B) This photograph shows transection of the pulmonary basal vein using an articulated endoscopic stapler. Note that adequate exposure of the vein periphery facilitates insertion of the stapler because the insertion angle of the stapler increases (extra-vessel exposure). A6: superior segmental artery; V6: superior segmental vein; Basal PA: the basal segmental artery; Basal PV: the basal segmental vein; Basal Br: the basal segmental bronchus.

Then, the affected bronchus was isolated and encircled, and the endoscopic stapler was inserted around the basal bronchus. We prefer a blue-colored 45-mm long stapler (Echelon Flex ${ }^{\mathrm{TM}}$ ENDOPATH ${ }^{\circledR}$ Stapler; Ethicon EndoSurgery, Inc., Cincinnati, Ohio, USA) for the approach to the basal segmental bronchus because it helps to ensure more consistent thickness of the targeted tissue (pre-compression). After the anesthesiologist inflated the left lung, the segmental bronchus was divided and the remaining
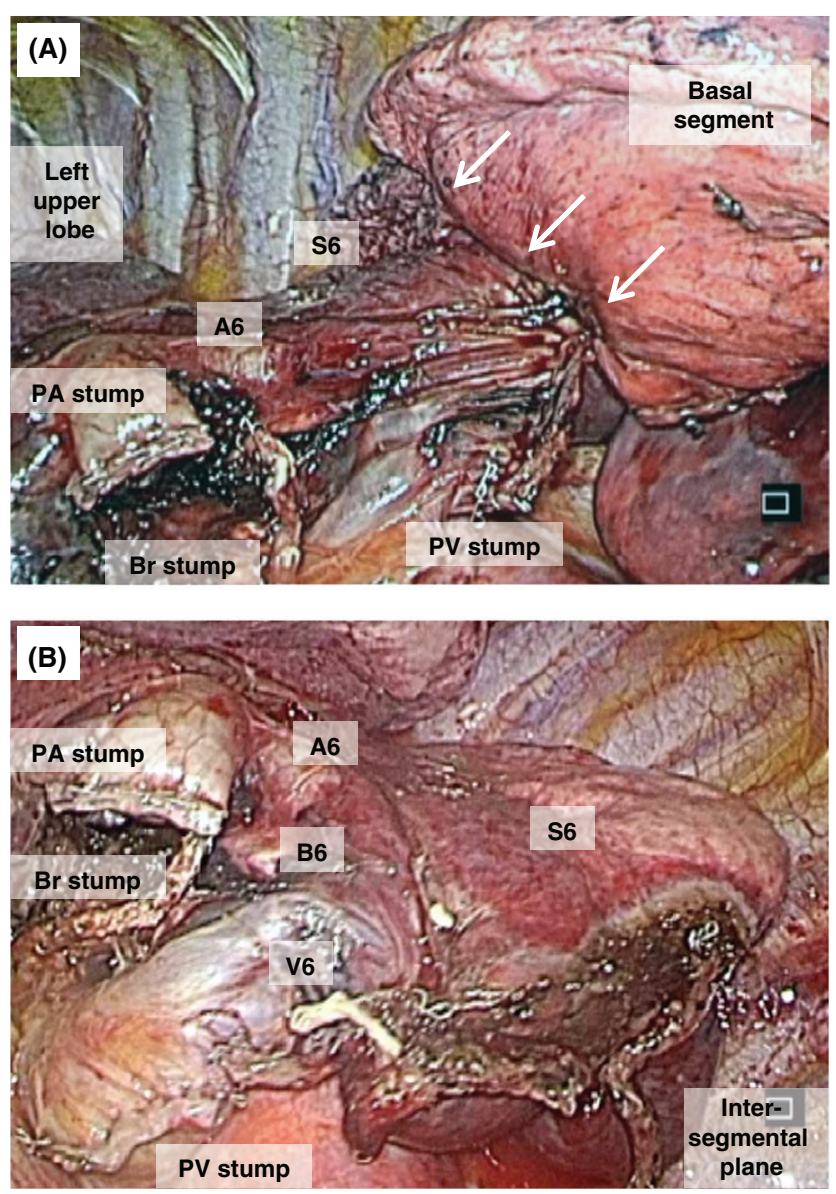

Fig. 3 (A) The inflation of the diseased segment (basal segment) allowed for the identification of the intersegmental plane (arrow). (B) The basal segment was removed using cautery and an endoscopic stapler. A6: superior segmental artery; B6: superior segmental bronchus; S6: superior segment of the left lower lobe; V6: superior segmental vein; PA stump: the stump of pulmonary artery; Br stump: the stump of bronchus; PV stump: the stump of pulmonary vein.

left lung was deflated. This maneuver kept the affected segment expanded, resulting in the identification of the intersegmental plane (Fig. 3A). Next, we cut the lung parenchyma 1-2 $\mathrm{cm}$ deep from the surface along the inflation-deflation line using electrocautery. With this line as a landmark, the remaining lung parenchyma was dissected with a stapler proximal to the incision and then distally (Fig. 3B).

Finally, the basal segment was removed in a plastic bag through the incision using the vacuum-packing method.7) The raw surface of the remaining lung was covered with an absorbable sealing material, polyglycolic acid felt (PGAF: Neoveil [sheet type]; Igaki Medical Planning 
Co., Ltd., Kyoto, Japan) to prevent air leakage. Lymph nodes 121 (lobar) and 13 (segmental) were also sampled. A 19-F silicone chest drain was placed through the incision. The operative time was $180 \mathrm{~min}$, and blood loss was less than $30 \mathrm{~mL}$.

The postoperative pathological findings demonstrated primary pulmonary adenocarcinomas in situ (11 and $10 \mathrm{~mm}$ ) and focal adenomatous hyperplasia (5 and $6 \mathrm{~mm})$ in the basal segment of the left lower lobe (pTisNOM0, staged as stage 0 [zero], complete resection). No analgesics were administered postoperatively. The chest tube was removed on postoperative day (POD) 2 and the patient was able to walk around the hospital ward. However, the patient developed acute respiratory distress syndrome (ARDS) on POD 3. The patient underwent intensive treatment, recovered, and was discharged 3 months after the onset of ARDS.

\section{Discussion and Conclusion}

Technically speaking, the most challenging aspect of uniportal VATS segmentectomy is identification of the intersegmental plane. Two previous reports of uniportal VATS anatomic segmentectomy have been published. Gonzalez-Riva, et al. ${ }^{2}$ and Wang, et al. ${ }^{3)}$ reported 17 and five cases, respectively, but they did not describe how the intersegmental plane was identified. It is possible that in these previous reports the authors were able to complete the uniportal procedure in a manner similar to conventional multi-port VATS. However, this seems unlikely because there was no angle for insertion of the endoscopic staples into the segmental plane. Therefore, in the case reported here, the intersegmental plane was dissected proximal to the incision and then distally. In addition, a 4- to 5-cm incision ${ }^{3)}$ seems too large to be considered uniportal VATS because such an incision is large enough to allow for direct visualization and anatomic pulmonary segmentation. ${ }^{8)}$

We generally do not use specialized instrumentation even when uniportal VATS is introduced. The use of conventional instruments for retraction and dissection during uniportal VATS is possible. However, additional procedural modifications are required to allow for simultaneous insertion of two or three instruments; we prefer to use the following unique techniques: ${ }^{9)}$

Shaft-on-shaft technique (Fig. 1B): We create a pivot point using a right-handed instrument during the shafton-shaft technique. Specifically, we hold the shaft of the right-handed instrument (e.g., scissors, an energy device, or a vacuum) against the shaft of a left-handed instrument (e.g., a grasper or a sponge stick) at the edge of the access incision.

One-hand encircling: The vessels and bronchi are encircled with a piece of silicone tape with one hand because excessive use of instruments crowds access to the incision. We use forceps to grasp the portion of the silicone tape distant from the tip in the long-axis direction and pass the forceps through the vessel. The tip of the silicone tape is then bent, and the silicone tape subsequently straightens. Finally, we release the silicone tape, pull the forceps, and grasp the tape again from the other side of the vessel.

One-hand exposure: We prefer to secure exposure of the operative field with one hand (the nondominant hand). We regrasp the more distal part of the initial position when the pulmonary parenchyma obstructs the visual field of the thoracoscope. Moving the entire lung parenchyma out to expose the visual field is not required during VATS. Exposing the direct path of the instruments is adequate.

When considered geometrically, ${ }^{10)}$ the approach for a target lung lesion is similar to a coaxial approach. That is, surgeons are working with their eyes and hands in the same plane (co-axial setup), much like open surgery and in contrast to three-port VATS (para-axial setup). Interestingly, the principles of conventional open surgery are helpful when performing uniportal VATS. However, the principles of conventional surgery may be difficult to implement for surgeons who have experience with only VATS.

Our criteria for uniportal VATS segmentectomy include stage IA lung cancers smaller than $2 \mathrm{~cm}$ in diameter, GGO, peripheral tumors, metastatic lung cancers, and the preservation of pulmonary function in cases of benign tumors. Specifically, we began performing the uniportal VATS major pulmonary resection without mediastinal lymph node dissection for the lower lobes because those presented less difficulty. The absence of lymph node involvement is always confirmed preoperatively by CT and PET-CT.

\section{Disclosure Statement}

The authors declare that we have received no financial support and have no relationships that may pose a conflict of interest. 


\section{References}

1) Gonzalez-Rivas D, Fieira E, Mendez L, et al. Singleport video-assisted thoracoscopic anatomic segmentectomy and right upper lobectomy. Eur J Cardiothorac Surg 2012; 42: e169-71.

2) Gonzalez-Rivas D, Mendez L, Delgado M, et al. Uniportal video-assisted thoracoscopic anatomic segmentectomy. J Thorac Dis 2013; 5: S226-33.

3) Wang BY, Tu CC, Liu CY, et al. Single-incision thoracoscopic lobectomy and segmentectomy with radical lymph node dissection. Ann Thorac Surg 2013; 96: 977-82.

4) Igai $H$, Kamiyoshihara M, Ibe T, et al. Single-incision thoracoscopic surgery for spontaneous pneumothorax using multi-degrees of freedom forceps. Ann Thorac Cardiovasc Surg 2014 Feb 4. [Epub ahead of print]

5) Gossot D. Technical tricks to facilitate totally endoscopic major pulmonary resections. Ann Thorac Surg 2008; 86: 323-6.
6) Kamiyoshihara M, Igai $\mathrm{H}$, Ibe $\mathrm{T}$, et al. Right superior mediastinal lymph node dissection in thoracoscopic surgery using a bipolar sealing device. Innovations (Phila) 2013; 8: 258-63.

7) Kamiyoshihara M, Igai H, Nagashima T, et al. A useful technique for specimen extraction from the thorax: the vacuum-packing method. Eur J Cardiothorac Surg 2012; 41: 1126-8.

8) Okada M, Tsutani Y, Ikeda T, et al. Radical hybrid video-assisted thoracic segmentectomy: long-term results of minimally invasive anatomical sublobar resection for treating lung cancer. Interact Cardiovasc Thorac Surg 2012; 14: 5-11.

9) Kamiyoshihara M, Igai H, Ibe T, et al. Initial report of uniportal video-assisted thoracoscopic lobectomy in Japan. Kyobu Geka 2014; 67: 540-3. (in Japanese with English abstract)

10) Bertolaccini L, Rocco G, Viti A, et al. Geometrical characteristics of uniportal VATS. J Thorac Dis 2013; 5: S214-6. 